\title{
A Corrective Strategy to Alleviate Overloading in Transmission Lines Based on Particle Swarm Optimization Method
}

\author{
Manoj Kumar Maharana* and K. Shanti Swarup \\ Department of Electrical Engineering, Indian Institute of Technology, Madras, Chennai 600 036, India \\ Received 20 May 2009; accepted 23 August 2009 \\ إستراتيجية تصحيحية لتففيف زيادة الثقل في خطوط التوصيل البعتهدة على طريقة فاعلية قثد الجزيئات \\ مانوج كومار مهارنا، ك. شانتي سواروب'

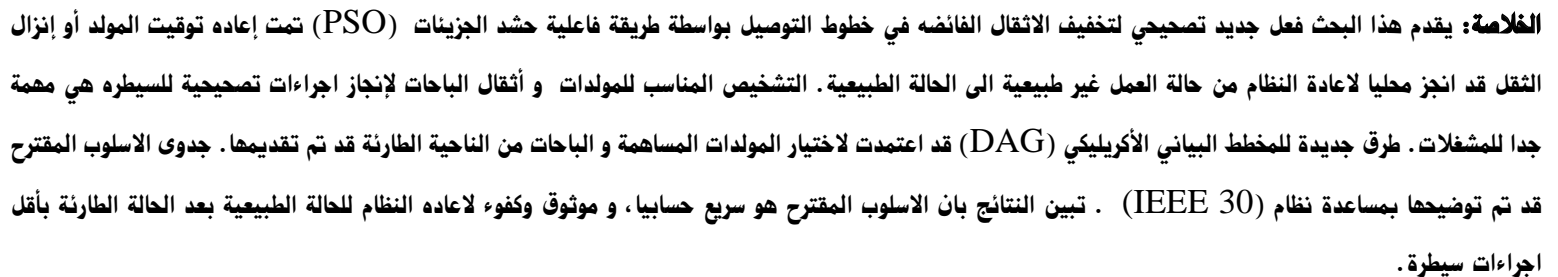 \\ الهزردات الهتاهية: مخطط بياني أكريليكي مباشر ، مولدات مساهمة، إستراتيجية سيطرة تصحيحية، فاعلية حشد الجزيئات، غعادة تشغيل الموده ، تعزيز الثقل.

\begin{abstract}
This paper presents novel corrective control actions to alleviate overloads in transmission lines by the Particle Swarm Optimization (PSO) method. Generator rescheduling and/or load shedding is performed locally, to restore the system from abnormal to normal operating state. The appropriate identification of generators and load buses to perform the corrective control action is an important task for the operators. A new Direct Acyclic Graph (DAG) technique for selection of participating generators and buses with respect to a contingency is presented. The effectiveness of the proposed approach is demonstrated with the help of the IEEE 30 bus system. The result shows that the proposed approach is computationally fast, reliable and efficient, in restoring the system to normal state after a contingency with minimal control actions.
\end{abstract}

Keywords: Direct Acyclic Graph, Participating Generators, Corrective Control Strategy, Particle Swarm Optimization, Generator Rescheduling, Load Shedding

\section{Nomenclatures}

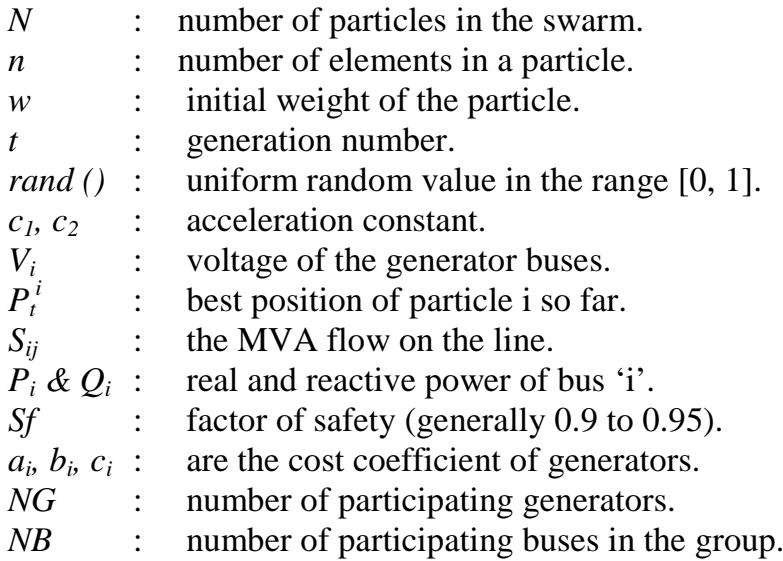


NL : number of lines in the group.

$P_{g i} \quad: \quad$ real power generated by the generator ' $\mathrm{i}$ '.

$P_{d} \& P_{L}: \quad$ are the total demand and loss of the system.

$G_{i j} \& B_{i j}$ : conductance and susceptance of the line ' $\mathrm{i}$ to $\mathrm{j}$ '.

$P_{t}^{i, g} \quad$ : global best position of particle in the population.

$K_{1}, K_{2}, K_{3}$ : the penalty factors (normally large positive real value).

\section{Introduction}

When a major power system disturbance occurs, protection and control actions are required to stop the power system's degradation. The aim of corrective control strategy is to avoid cascading outages or system collapse, and to maintain system reliability. The selection of generators and load buses for corrective control action is a crucial task for the system operator. A fast identification of the participating generators, load buses and a proper control action are essential for secure and reliable operation of a power system. Under this condition, a minimum number of control actions like rescheduling of generators/load shedding for the participating generators and loads are efficient for the affected power system.

In literature many methods for transmission line overload alleviation via corrective strategies have been report ed. Alleviation of line overloads by generator rescheduling/load shedding based on Redial Basic Function (RBF) neural network is reported by Ram et al. (2007). Conjugate gradient technique to minimize the line overloads in conjunction with the local optimization is given by Shandilya et al. (1993). There are several publications available that describe direct methods of line overload alleviation using generation rescheduling and load shedding (Talukdar et al. (2005); Medicheral et al. (1981); Chan et al. (1979) and Christie et al. (2000). In these methods the system operator has no choice over the selection of the generators or tagged buses to alleviate the overloads.

Relative-Electrical-Distance-based (RED-based) real power rescheduling for the participating generators to alleviate overload of lines is proposed by Yesuratnam et al. (2007), whereas multi-objective-PSO-based generator rescheduling/load shedding for alleviation of overload in a transmission network is proposed by Hazra et al.; (2007). Sensitivity-optimized-based participating generator selection and alleviating overloads by rescheduling generators is proposed by Dutta et al.; (2008). In this type of control, all the generators in a system may be divided into two groups, but in practical cases some generators do not supply power to the congested lines. In such cases, all the generators are handled unnecessarily, increasing the complexity of the control strategy. The Optimal Power Flow (OPF) technique is the most accurate method for overload alleviation. However, OPF calculation is computationally expensive and time-consuming.

The main intent of the paper is to propose a technique to identify the participating generators for corrective control actions. In this DAG is used to identify the participat ing generators and load buses for generator rescheduling/load shedding. With respect to the contingency, the participating generators are classified into two groups based on the power flow directions. Generation in one group of generators is increased while in the other group it is decreased. Generators which are contributing to the contigency line (generator flows contributing to the contingency line) to the contingency line identified as the Generator Decrease (GD) group and the generators which are not contributing to the contingency line are categorized as the Generator Increase (GI) group. The corrective control strategy is modeled as an optimization problem. From the literature it has been found that the Particle Swarm Optimization (PSO) method proposed by Kennedy and Eberhart (1995) is robust and fast in solving non-linear, non-differentiable problems. The corrective control action for overload-alleviation is a PSO-based-generatorrescheduling and/or load-shedding method applied to the GI and GD groups. The proposed corrective control action provides an optimal solution of generator-rescheduling and load-shedding which would bring back the system to normal state.

\section{Graph Theoretic Approach}

In a power system, all generators do not supply power to all loads. The generators which supplying power to a particular load can be identified easily by graph theory. Graph theory converts the entire power system into a unidirectional hierarchical structure, based on the power flow contribution from the generators to the loads. Graph theory organizes the buses and lines of the network into a homogeneous group according to the concept of 'reach of a generator', 'generator area' and 'links'. The homogeneous group is called the direct acyclic graph (DAG) and it is unidirectional in nature.

\subsection{Directed Acyclic Graph (DAG)}

A graph is a set of nodes and a set of edges. A cycle is a path with the same node at the beginning and the end. An acyclic graph is a graph with no path that starts and ends at the same node. A directed acyclic graph (DAG) contains no cycles; this means that if there is a route from node 'a' to node 'b', then there is no way back.

\subsubsection{Reach of a Generator (ROG):}

The reach of a generator is defined as the set of buses which are reached by power produced by that generator. 
Power from a generator reaches a particular bus if it is possible to find a path through the network from the generator to the bus for which the direction of travel is always consistent with the direction of the flow as computed by a power flow program or a state estimator proposed by Bialek; (1996) and Kirschen et al. (1997). For large systems, the reach of a generator (ROG) can be determined using the algorithm, explained in the flowchart shown in Fig. 1.

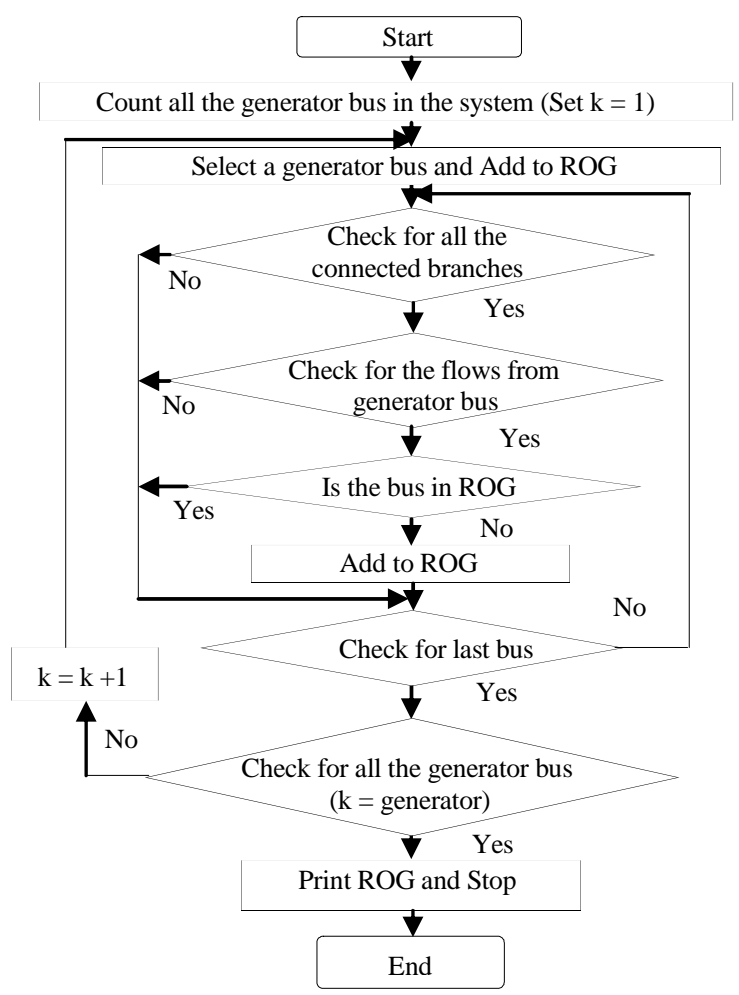

Figure 1. Flow Chart for Reach of a Generator (ROG)

\subsubsection{Generator Area (GA):}

The generator area is defined as a set of contiguous buses supplied by the same generator. Unconnected sets of buses supplied by the same generator are treated as separate generator area. A bus therefore belongs to one and only one generator area. The rank of generator area is defined as the number of generators supplying power to the buses. It can never be lower than one or higher than the number of generators in the system. For networks of a more realistic size, the generator area can be determined using the algorithm which is explained in the flowchart shown in Fig. 2.

\subsubsection{Links:}

Having divided the buses into generator areas, each branch is either internal (ie. it connects two buses which are part of the same generator area) or external (ie. it connects two buses which are part of different generator area) to a generator area. One or more external branches connecting the different generator area will be called a link. It is very important to note that the actual flows in all the branches of a link are all in the same direction. Furthermore, this flow in a link is always from a generator areas of rank $\mathrm{N}$ to a generator area of rank $\mathrm{M}$ where $\mathrm{M}$ is always strictly greater than $\mathrm{N}$.

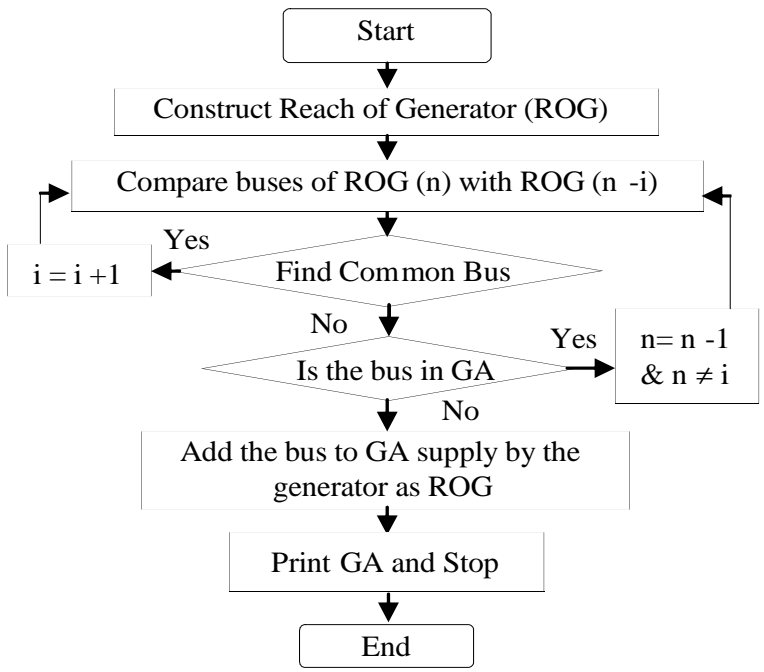

Figure 2. Flowchart for Generator Generator Area (GA)

The construction of DAG with the help of ROG, GA and links will be understood easily by an example given below. For example consider a 6-bus 11-line system. Figure 3(a) shows the real power flows from generator to load. By definition the ROG of generator $\mathrm{G}_{1}$ encompasses all the buses generator $G_{2}$ includes buses 2, 3, 4, 5, 6 and the ROG of generator $G_{3}$ includes buses 3, 5 and 6 . Bus 1 , which is supplied by generator $G_{1}$ only comes under Generator area $\mathrm{GA}_{1}$ and the rank is one as only $\mathrm{G}_{1}$ power to it. Similarly buses 2 and 4 come under generator area $\mathrm{GA}_{2}$ and the rank is 2 as power is supplied by both generator $G_{1}$ and $G_{2}$. Buses 3,5 and 6 come under generator area $\mathrm{GA}_{3}$ and these buses are supplied power from all the three generators, so their rank is 3 . Lines 1-2 and 1-4 are links between generator areas $\mathrm{GA}_{1}$ and $\mathrm{GA}_{2}$, whereas lines 2-3, 2-5, 2-6 and 4-5 are links between generator area $\mathrm{GA}_{2}$ and $\mathrm{GA}_{3}$. The line 1-5 is the link between generator areas $\mathrm{GA}_{1}$ and $\mathrm{GA}_{3}$. The DAG for a six-bus system is shown in Fig. 3(b).

\section{Particle Swarm Optimization (PSO)}

PSO is a simple and efficient population-based optimization method proposed by Kennedy and Eberhart (1995). PSO is motivated by social behavior of organisms such as fish-schooling and bird-flocking. In PSO, potential solutions called particles fly around in a multidimensional problem space. A population of particles is called a swarm. Each particle in a swarm flies in the search space towards the optimum or a quasi-optimum solution based on its own experience, experience of nearby particles, and 


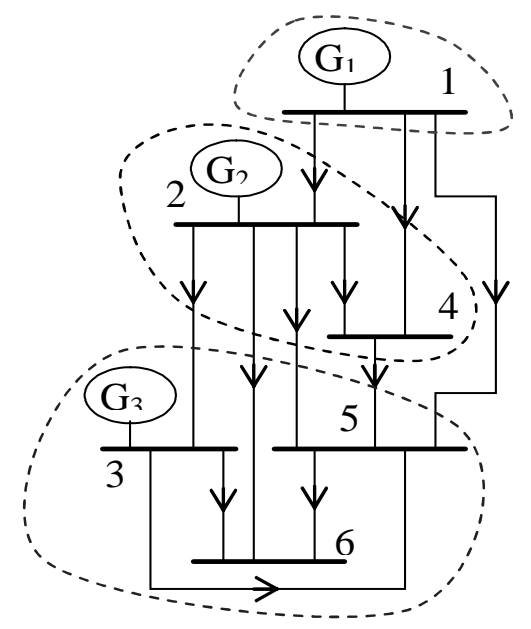

Figure 3. (a) Flow diagram of six bus system

global best position among particles in the swarm. Let us define a search space $\mathrm{S}$ as $\mathrm{n}$-dimension while the swarm consists of $\mathrm{N}$ particles. At time t, each particle $\mathrm{i}$ has its position defined by $X_{t}^{i}=\left\{x_{1}^{i}, x_{2}^{i}, \ldots ., x_{n}^{i}\right\}$ and a velocity defined by $V_{t}^{i}=\left\{v_{1}^{i}, v_{2}^{i}, \ldots ., v_{n}^{i}\right\}$ in variable space $\mathrm{S}$. The position and velocity of each particle change with time. The velocity and position of each particle in the next generation (time step) can be calculated as

$$
\begin{aligned}
V_{t+1}^{i} & =w \times V_{t}^{i}+c_{1} \times \operatorname{rand}() \times\left(P_{t}^{i}-X_{t}^{i}\right) \\
& +c_{2} \times \operatorname{rand}() \times\left(P_{t}^{i, g}-X_{t}^{i}\right) \\
X_{t+1}^{i} & =X_{t}^{i}+V_{t+1}^{i} \quad i=1,2, \ldots ., N
\end{aligned}
$$

The acceleration constant $c_{1}$ called cognitive parameter pulls each particle towards local best position whereas constant $\mathrm{c}_{2}$ (called social parameter) pulls the particle towards a global best position. Usually $c_{1}$ and $c_{2}$ range from 0 to 4 given in PSO Tutorial. [Online].

The inertia weight $w$ is an important factor for the PSO's convergence. It is used to control the impact of the previous history of velocities on the current velocity. A large inertia weight factor facilitates global exploration (ie. searching of a new area) while a small weight factor facilitates local exploration. Therefore, it is wise to choose a large weight factor for initial iterations and gradually reduce the weight factor in successive iterations given by Bhaskar et al. (2008). This can be done by using

$$
w=w_{\max }-\frac{w_{\max }-w_{\min }}{\text { iter }_{\max }} \times \text { iter }
$$

where $w_{\max }$ and $w_{\min }$ are maximum and minimum weight, respectively, iter is iteration number, and $i t e r_{\max }$ is maximum iteration allowed.

With no restriction on the maximum velocity $\left(V_{\max }\right)$ of the particles, velocity may move towards infinity. If $V_{\max }$

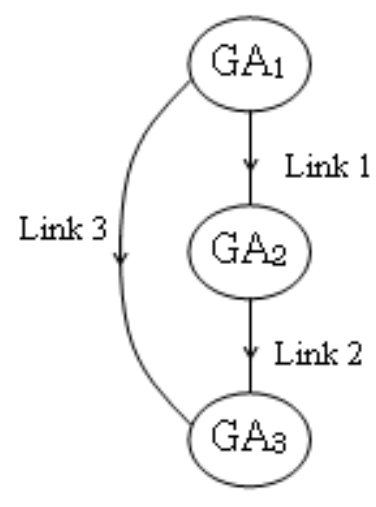

(b) DAG of six bus system

is very low, the particle may not explore sufficiently, and if $V_{\max }$ is very high, it may oscillate about optimal solution. Velocity clamping effect has been introduced to avoid the phenomenon of "swarm explosion". In the proposed method, velocity is controlled within a band as

$$
V_{\text {max }, t}=V_{\text {max }}-\frac{V_{\text {max }}-V_{\text {min }}}{\text { iter }_{\text {max }}} \times \text { iter }
$$

where $V_{\text {max,t }}$ is maximum velocity at generation $\mathrm{t}$, and $V_{\max }$ and $V_{\min }$ are initial and final velocity, respectively.

\section{Mathematical Formulation for Corrective Control Strategy}

The corrective control strategy by generator rescheduling/load shedding has been divided into two groups (GD and GI) of optimization problems below.

\subsection{Modeling for Generator Decrease (GD) Group}

In the Generator Decrease group, the goal is to reduce the generation with respect to load such that the bus voltage constraints are within the limits. This problem can be solved by classical economic load dispatch with lineflow and voltage limits as constraints. The objective of the constrained economic dispatch problem (ie. voltage and line flow constraints) is to determine the most economic loading of the generators such that the load demand in the GD group are within their limits. The objective is to determine the optimal set of generation $P_{g i}(i=1,2, \ldots N G)$ so as to minimize the total cost of generation " $F_{t}$ " given by

$$
\text { Minimize } f(x) \cong F_{t}=\sum_{i=1}^{N G}\left(a_{i} P_{g i}^{2}+b_{i} P_{g i}+c_{i}\right)
$$

Subject to Equality constraints

$$
g(x)=0 \cong \sum_{i=1}^{N G}\left(P_{g i}\right)-P_{d}-P_{L}=0
$$


Inequality constraints

$$
h(x) \leq 0 \cong\left[\begin{array}{l}
P_{g i}^{\min } \leq P_{g i} \leq P_{g i}^{\max } \\
V_{i}^{\min } \leq V_{i} \leq V_{i}^{\max } \\
S_{i j} \leq S_{i j}^{\max }
\end{array}\right.
$$

Fitness function $F^{*}$ used in PSO for this group is formulated including all the constraints as follows.

$$
\begin{aligned}
F_{t}^{*} & =F_{t}+K_{1} \sum_{i=1}^{N B}\left(V_{L i}-V_{L i}^{L i m}\right)^{2}+K_{2} \sum_{j=1}^{N L}\left(S_{i j}-S_{i j}^{\max }\right)^{2} \\
& +K_{3}\left(P_{\text {Slack }}-P_{\text {Slack }}^{\text {Lim }}\right)^{2}
\end{aligned}
$$

\subsection{Modeling for Generator Increase (GI) Group}

The aim of the Generator Increase group is to increase the generation within the generator limits so as to meet the demand, and if not possible, switch to load-shedding. As generation increases in this group, there may be an overload in some of the lines. Alleviation of overloads in the GI group can be formulated as an optimization problem as follows:

The objective function

$$
f(x)=0 \cong \sum_{i j \in \text { all }}\left(S_{i j}-S_{i j}^{\max } \times S f\right)^{2}=0
$$

Subject to Equality constraints:

$$
g(x)=0 \cong\left[\begin{array}{c}
P_{i}-\sum_{j \in \text { all }} G_{i j} v_{i}^{2}-v_{i} v_{j}\left[G_{i j} \cos \left(\theta_{i}-\theta_{j}\right)\right. \\
\left.+B_{i j} \sin \left(\theta_{i}-\theta_{j}\right)\right]=0 \\
Q_{i}+\sum_{j \in \text { all }} B_{i j} v_{i}^{2}+v_{i} v_{j}\left[B_{i j} \cos \left(\theta_{i}-\theta_{j}\right)\right. \\
\left.-G_{i j} \sin \left(\theta_{i}-\theta_{j}\right)\right]=0
\end{array}\right.
$$

Inequality constraints:

$$
h(x) \leq 0 \cong\left[\begin{array}{l}
P_{i}^{\min } \leq P_{i} \leq P_{i}^{\max } \\
Q_{i}^{\min } \leq Q_{i} \leq Q_{i}^{\max } \\
V_{i}^{\min } \leq V_{i} \leq V_{i}^{\max }
\end{array}\right.
$$

The fitness function $F^{*}$ used in PSO for this group is formulated as follows:

$$
F_{t}^{*}=\sum_{j=1}^{N L}\left(S_{i j}-S_{i j}^{\max } \times S f\right)^{2}+K_{1} \sum_{i=1}^{N B}\left(V_{L i}-V_{L i}^{L i m}\right)^{2}
$$

\section{Algorithm for Proposed Corrective Cont- rol Strategy}

To alleviate line overloads due to contingency of one or more lines in the system, the following sequence of control actions is expected from the operator.

1. Decrease the bus power injections at the sending end bus of the contingency line. This is incorporated by decreasing the generation at this bus and/or at the buses feeding power to it.

2. Maintain the bus power injections constant at the receiving end bus of the contingency line by increasing the generation at this bus and/or at the buses feeding power to it.

3. If the load demand is not met satisfying all the line constraints, then curtail the load at the receiving-end participating loads to which power is being fed from this bus.

Based on Powerflow/state estimation results the pre and post-contingency DAG are constructed, and GD and GI groups are identified, by comparing the pre-and-postcontingency generator areas. The generator rescheduling and/or load shedding optimization problems for the GD and GI group are solved by a PSO technique. Adjustment of generation and loads for the participating generators and load buses obtained from the PSO technique are the corrective control actions for the alleviation of overloads. In the GD group, adjust the generation to a load within minimum generation cost, in the GI group, adjust the generation and load such that there are no overloads in any lines in this group. The implementation procedure of the proposed algorithm is given below.

Step 1: Initialize randomly the individual of the population according to the limits of each generating unit (except a slack bus) including individual dimensions, searching points and velocities. The new velocity strategy equation is formulated and the maximum and minimum velocity limits of each individual are calculated using (13) and (14)

$$
\begin{aligned}
& V_{d}^{\max }=\left(\frac{P_{d}^{\max }-P_{d}^{\min }}{2}\right) \times \beta \\
& V_{d}^{\min }=-\left(\frac{P_{d}^{\max }-P_{d}^{\min }}{2}\right) \times \beta
\end{aligned}
$$

Where $P_{d}^{\max }=\sum_{i=1}^{n} P_{i}^{\max }$ and $P_{d}^{\min }=\sum_{i=1}^{n} P_{i}^{\min }$ $\mathrm{i}=1,2, \ldots \mathrm{n}$ (number of generators) and $\hat{\mathrm{a}}=0.01 \mathrm{a}$ smaller value for smooth convergence.

Step 2: Compute the slack bus generator vector, losses and line flows using Newton-Raphson load-flow method for the above generators. 
Step 3: To account for slack unit limit violation, and voltage limit violation, the total operating cost is augmented by non-negative penalty terms $\mathrm{K}_{1}, \mathrm{~K}_{2}$ and $\mathrm{K}_{3}$. Calculate augmented cost $F^{*}{ }_{t}$ using (8) for GD group and (12) for GI group.

Step 4: Among the population, the minimum augmented fuel cost value is taken as the best value. The best-augmented fuel cost value in the population is denoted as the Gbest. Remaining individuals are assigned as the Pbest.

Step 5: Modify the velocity $\mathrm{V}$ of each individual real power-generating unit $\mathrm{P}_{\mathrm{gi}}$ using (1).

Step 6: Check the limits on velocity using (15)

$$
\begin{aligned}
& \text { If } V_{i d}^{(t+1)}>V_{d}^{\max } \text {, then } V_{i d}^{(t+1)}=V_{d}^{\max } \\
& \text { If } V_{i d}^{(t+1)}<V_{d}^{\min } \text {, then } V_{i d}^{(t+1)}=V_{d}^{\min }
\end{aligned}
$$

Step 7: Modify the member position of each individual $\mathrm{P}_{\text {gi }}$ using (16).

$$
P g_{i d}^{(t+1)}=P g_{i d}^{(t)}+V_{i d}^{(t+1)}
$$

Step 8: $\quad P g_{\text {id }}^{(t+1)}$ must satisfy the capacity limits of the generators and are given by

$$
\begin{aligned}
& \text { If } \operatorname{Pg}_{i d}^{(t+1)}>P g_{i d}^{\max } \text {, then } \operatorname{Pg}_{i d}^{(t+1)}=P g_{i d}^{\max } \\
& \text { If } \operatorname{Pg}_{i d}^{(t+1)}<P g_{i d}^{\min } \text {, then } \operatorname{Pg}_{i d}^{(t+1)}=P g_{i d}^{\min }
\end{aligned}
$$

Step 9: Modified member positions in step 8 are taken as the initial value for the N-R load-flow method. Compute slack bus power and line-flows using the N-R load flow method.

Step 10: Calculate the augmented fuel cost using (8) for the GD group and (12) for the GI group. Gbest and Pbest values are assigned. If the Gbest value is better than Gbest value in Step 4 then current value is set to Gbest. If the present Pbest value is better than the Pbest value in Step 4, current value is set to Pbest.

Step 11: In the GD group if the iteration reaches the maximum go to Step 13, otherwise go to Step 4 and the Gbest and Pbest values obtained in Step4 are replaced by the latest Gbest and Pbest values acquired in Step 10. In the GI group if the iteration reaches the maximum and the solution does not converge, then go to step 12.

Step 12: Reduce the load using the load-reduction factor given in Eq. (18) and jump to Step 4 after replacing Gbest and Pbest values with the latest values obtained in Step 10.
Load Reduction Factor $(\mathrm{LRF})=$

Net Load at overload bus - Allowable Po wer to the bus

$$
\text { Total MVA Load }
$$

Present modified load $=(1$. LRF $x$ Initial MVA load at the bus

Step 13: The latest Gbest value generated by the individual is the optimal generation for each unit, which is obtained by satisfying the reduced loads and all constraints in GI the group.

\section{Simulation and Results}

To verify the effectiveness of the proposed corrective control strategy, simulation was carried out on the IEEE 30 bus power system. The simulation was done in a $2.66 \mathrm{GHz}$ Pentium IV, $512 \mathrm{MB}$ RAM personal computer. Cost Coefficients and MW limits of the generators are given in Appendix A. The selection of contingency cases was considered randomly. The upper and lower limits of load bus voltages were taken as 1.05 p.u. and 0.95 p.u. respectively. The generator bus voltages were fixed to their specified values. Lineloading (MVA) limits of $125 \%$ of base case were considered. In the PSO-based optimization method, a population size of 10 with number of iterations limited to a maximum of 50 was taken. An increases in population size provides better optimal results sacrificing more computation time. PSO parameters $\mathrm{c}_{1}=2.0, \mathrm{c}_{2}=2.1, \mathrm{w}_{\max }=0.9, \mathrm{w}_{\min }=0.4$ were selected from Hazra et al.; (2007) and Bhaskar et al.; (2008). For each test case, 50 independent trials were carried out. An acceptable variation from $\pm 1 \%$ to $\pm 10 \%$ is observed in few worst cases among the 50 trials.

\subsection{Case 1: Outage of line 4-12 of IEEE 30 Bus}

The buses occupied by the generator areas for the base case and the changes of buses due to outage of lines 4-12 are given in Table-1. The base-case power-flow diagram indicating different generator areas is given in Fig. 4.

The base case Direct Acyclic Graph (DAG) acquired from Table 1 is shown in Fig. 5(a). The bus numbers occupied by the generator areas $\mathrm{GA}_{11}, \mathrm{GA}_{13}$ and $\mathrm{GA}_{\mathrm{Com}}$ are altered, there is no change of buses in the generator areas $G_{1}, G A_{2}, G A_{5}$, and $G_{8}$ after the outage of line 412 as given in Table 1 . Before outage the flows in the lines 4-12 are supplied from $\mathrm{GA}_{1}$ and $\mathrm{GA}_{2}$. The generator areas $\mathrm{GA}_{1}$ and $\mathrm{GA}_{2}$ are considered as GD group where as generator areas $\mathrm{GA}_{8}, \mathrm{GA}_{11}, \mathrm{GA}_{13}$ and $\mathrm{GA}_{\mathrm{Com}}$ are considered as the GI group as shown in Fig. $5(\mathrm{~b})$.

The nature of PSO convergence characteristics for the GD and GI groups is shown in Fig. 6. The convergence time for the GD group varies from $1.93 \mathrm{sec}$ to $2.2 \mathrm{sec}$ and for the GI group it varies from $9.1 \mathrm{sec}$ to $9.8 \mathrm{sec}$ respectively. 
Table 1. Buses occupied by generator areas for IEEE 30 bus system

$\begin{array}{cccc}\begin{array}{c}\text { Sl. } \\ \text { No. }\end{array} & \begin{array}{c}\text { Generator } \\ \text { Area }\end{array} & \begin{array}{c}\text { Bus Numbers } \\ \text { occupied } \\ \text { (base case) }\end{array} & \begin{array}{c}\text { Bus Numbers } \\ \text { occupied } \\ \text { (after outage } \\ \text { of line 4-12) }\end{array} \\ 1 & \mathrm{GA}_{1} & 1,3 & 1,3 \\ 2 & \mathrm{GA}_{2} & 2,4 & 2,4 \\ 3 & \mathrm{GA}_{3} & 6,7,8,27-30 & 6,7,8,27-30 \\ 4 & \mathrm{GA}_{4} & 12-16,18,23 & 13 \\ 5 & \mathrm{GA}_{5} & 5 & 5 \\ 6 & \mathrm{GA}_{6} & 9,10,11,20- & 9,10,11,16- \\ & & 22 & 26 \\ 7 & \mathrm{GA}_{7} & 17,19,24-26 & 12,14,15\end{array}$

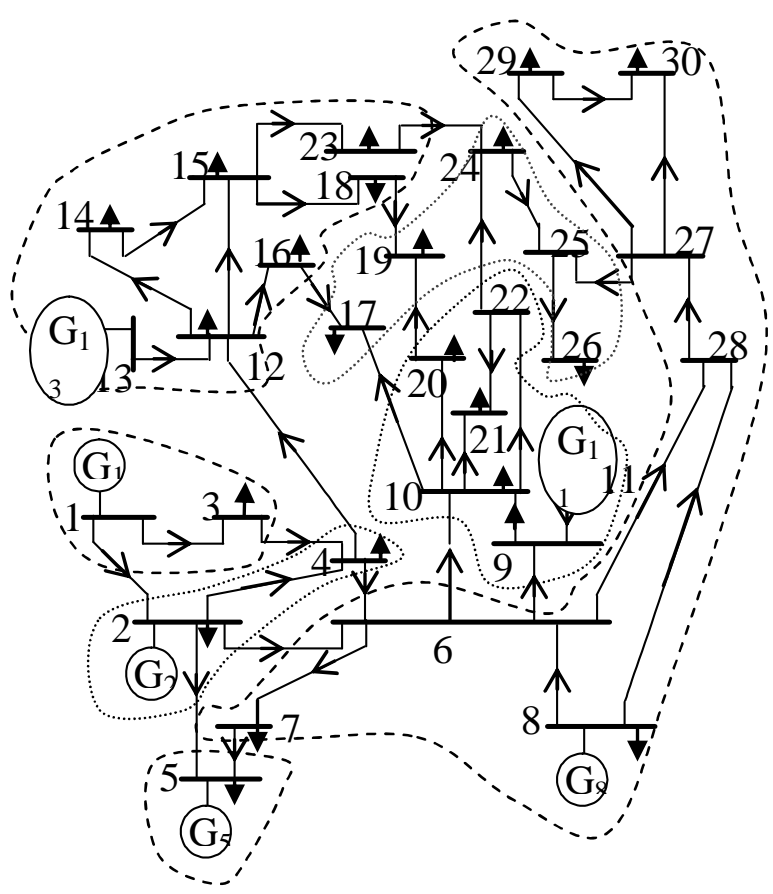

Figure 4. Base case flow diagram indicating generator area of IEEE 30 bus system

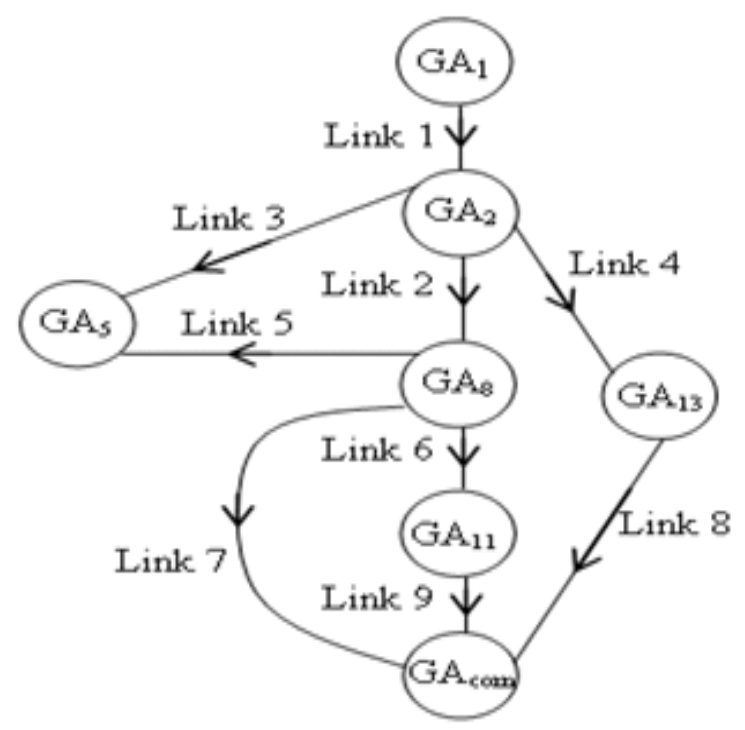

Figure 5. (a) Base case DAG for IEEE 30 bus system

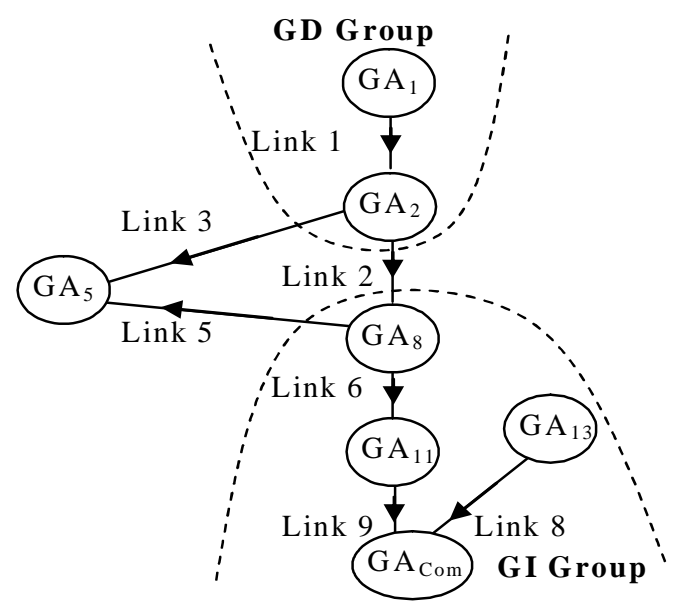

Figure 5. (b) DAG after outage of line 4-12

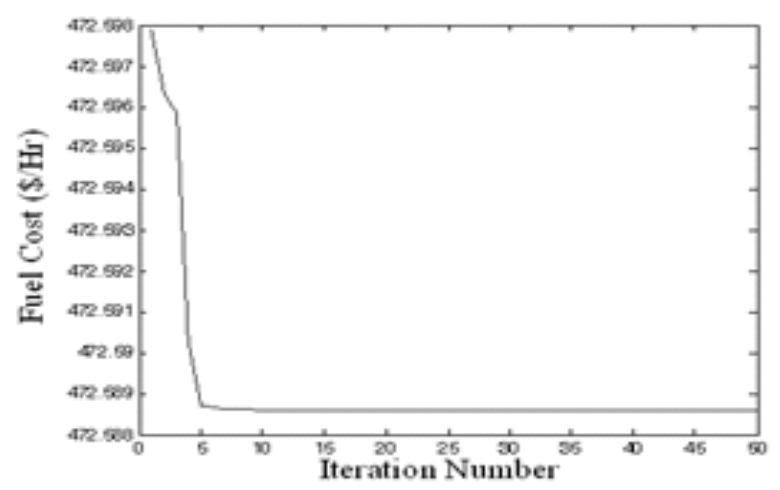

(a) GD group

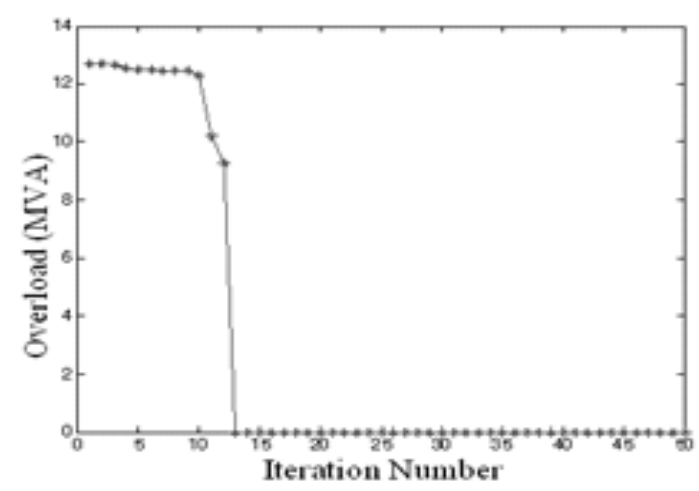

(b) GI group

Figure 6. PSO convergence characteristics for outage of line 4-12

Table- 2 shows the results of the corrective control strategy of generator rescheduling/load shedding for the outage of line 4-12. It can be observed from Table-2 that the line flows after the occurrence of contingency (B) exceeds the MVA limits (A). The line overloading is removed by rescheduling generators $1,2,8,11,13$ and a 30.13 MVA of load shedding, shared by load buses 12, 14-19, 23 and 24 respectively. The post-contingency flows (C) are within the MVA limits (A) after the control strategy as seen from Table-2. The pre-contingency and post-contingency voltage magnitudes after corrective control action are given in Table 3. From Table 3 it is observed that all load bus voltages are with in the voltage limits. 
Table 2. Simulation results for outage of line 4-12 of IEEE 30 bus system

\section{Overload Condition}

$\begin{array}{rcc}\text { Line } & \text { (A) } & \text { (B) } \\ \text { 4-12 } & \text { Max } & \text { Contin } \\ \text { out } & \text { Cap. } & \text {-gency }\end{array}$

Lines MVA $\quad$ MVA

4-6 $\quad 48.23 \quad 62.68$

$6-9 \quad 20.86 \quad 30.13$

$6-10 \quad 14.79 \quad 20.34$

$9-10 \quad 41.15 \quad 47.67$

$12-13 \quad 23.38$

$16-17 \quad 5.11$

$18-19 \quad 3.57$

$19-20$

$10-20$

$10-17$

$22-24$

$23-24$

$24-25$

$25-27$

28-27

$6-28$

13.77

$12.19 \quad 16.44$

$8.56 \quad 18.92$

$8.98 \quad 9.85$

$3.19 \quad 5.91$

$1.88 \quad 5.46$

$\begin{array}{ll}4.32 & 8.28\end{array}$

$21.84 \quad 22.17$

$16.33 \quad 17.03$

\section{Corrective Control Strategies}

\begin{tabular}{|c|c|c|c|c|c|c|c|}
\hline \multicolumn{3}{|c|}{ Generation } & \multicolumn{3}{|c|}{ Load } & \multirow{2}{*}{$\begin{array}{l}(\mathrm{C}) \\
\text { Post } \\
\text { cont. } \\
\text { Flow }\end{array}$} & \multirow[b]{2}{*}{ Remarks } \\
\hline \multicolumn{2}{|c|}{$\begin{array}{c}\text { Pre - } \\
\text { contingency }\end{array}$} & \multirow{2}{*}{$\begin{array}{c}\text { Control } \\
\text { action } \\
\text { MW }\end{array}$} & \multicolumn{2}{|c|}{$\begin{array}{c}\text { Pre - } \\
\text { contingency }\end{array}$} & \multirow{2}{*}{$\begin{array}{l}\text { Control } \\
\text { action } \\
\text { MVA }\end{array}$} & & \\
\hline Bus & MW & & Bus & MVA & & MVA & \\
\hline $1^{*}$ & 138.69 & 105.58 & 2 & 25.14 & 25.14 & 46.35 & 30.13 \\
\hline $2 *$ & 57.56 & 59.17 & 3 & 2.68 & 2.68 & 14.64 & MVA loac \\
\hline 5 & 24.56 & 24.56 & 4 & 7.77 & 7.77 & 12.09 & shedding \\
\hline 8* & 35.00 & 37.12 & 10 & 6.14 & 6.14 & 35.22 & and there \\
\hline $11^{*}$ & 17.93 & 22.41 & $12 *$ & 13.48 & 7.81 & 20.73 & are no \\
\hline $13^{*}$ & 16.91 & 18.24 & $14 *$ & 6.40 & 3.71 & 2.17 & overload \\
\hline & & & $15^{*}$ & 8.57 & 4.69 & 0.78 & in any \\
\hline & & & $16^{*}$ & 3.94 & 2.28 & 6.61 & lines \\
\hline & & & $17^{*}$ & 10.71 & 6.21 & 9.05 & \\
\hline & & & $18 *$ & 3.33 & 1.93 & 8.28 & \\
\hline & & & $19 *$ & 10.09 & 5.85 & 8.16 & \\
\hline & & & 20 & 2.31 & 2.31 & 1.12 & \\
\hline & & & 21 & 20.77 & 20.77 & 1.18 & \\
\hline & & & $23 *$ & 3.57 & 2.07 & 4.16 & \\
\hline & & & $24 *$ & 10.98 & 6.36 & 18.17 & \\
\hline & & & 26 & 4.18 & 4.18 & 12.88 & \\
\hline
\end{tabular}

The '*' indicates the alteration of generation and loads as corrective control action at that bus

Table 3. Pre and post contingency voltage magnitude

Pre Contingency Bus Voltage

$\begin{array}{cccc}\text { Sl. No. } & \text { Voltage (pu) } & \text { Sl. No. } & \text { Voltage (pu) } \\ 1 & 1.050 & 16 & 1.047 \\ 2 & 1.045 & 17 & 1.043 \\ 3 & 1.023 & 18 & 1.031 \\ 4 & 1.017 & 19 & 1.028 \\ 5 & 1.010 & 20 & 1.033 \\ 6 & 1.014 & 21 & 1.036 \\ 7 & 1.004 & 22 & 1.036 \\ 8 & 1.010 & 23 & 1.030 \\ 9 & 1.053 & 24 & 1.024 \\ 10 & 1.048 & 25 & 1.019 \\ 11 & 1.082 & 26 & 1.002 \\ 12 & 1.060 & 27 & 1.025 \\ 13 & 1.071 & 28 & 1.009 \\ 14 & 1.045 & 29 & 1.005 \\ 15 & 1.041 & 30 & 0.993\end{array}$

Post Contingency Voltage (After control action)

Sl. No. Voltage (pu) Sl. No. Voltage (pu)

$\begin{array}{cccc}1 & 1.050 & 16 & 1.046 \\ 2 & 1.045 & 17 & 1.048 \\ 3 & 1.039 & 18 & 1.046 \\ 4 & 1.036 & 19 & 1.047 \\ 5 & 1.010 & 20 & 1.050 \\ 6 & 1.032 & 21 & 1.049 \\ 7 & 1.015 & 22 & 1.049 \\ 8 & 1.035 & 23 & 1.042 \\ 9 & 1.045 & 24 & 1.039 \\ 10 & 1.044 & 25 & 1.037 \\ 11 & 1.082 & 26 & 1.019 \\ 12 & 1.048 & 27 & 1.043 \\ 13 & 1.071 & 28 & 1.029 \\ 14 & 1.049 & 29 & 1.024 \\ 15 & 1.048 & 30 & 1.013\end{array}$

Table 4. Comparison result for congestion management of lines 1-2 and 2-6

\begin{tabular}{ccc} 
Generator & \multicolumn{2}{c}{ Generator Rescheduling (MW) $\mathbf{\Delta P}$} \\
Number & Result Reported on Dutta et al. (2008) & Proposed Approach \\
1 & -59.0 & -55.59 \\
2 & 19.9 & 18.70 \\
5 & 13.0 & 18.30 \\
8 & 6.0 & 4.60 \\
11 & 6.5 & 9.20 \\
13 & 7.0 & Idle \\
Total (MW) & 111.4 & 106.39
\end{tabular}


The Journal of Engineering Research Vol. 7, No. 1, (2010) 31-41

Table 5. Generators statistical performance for $\mathbf{5 0}$ trials

Participating Generators for outage of line 4-12

$\begin{array}{cccc}\text { Generator Number } & \text { Max (MW) } & \text { Min (MW) } & \text { Best (MW) } \\ 1 \text { (GD) } & 109.56 & 104.43 & 107.58 \\ 2 \text { (GD) } & 61.71 & 57.55 & 59.77 \\ 8 \text { (GI) } & 39.06 & 30.78 & 34.43 \\ 11 \text { (GI) } & 24.89 & 19.86 & 22.33 \\ 13 \text { (GI) } & 21.44 & 16.39 & 18.69\end{array}$

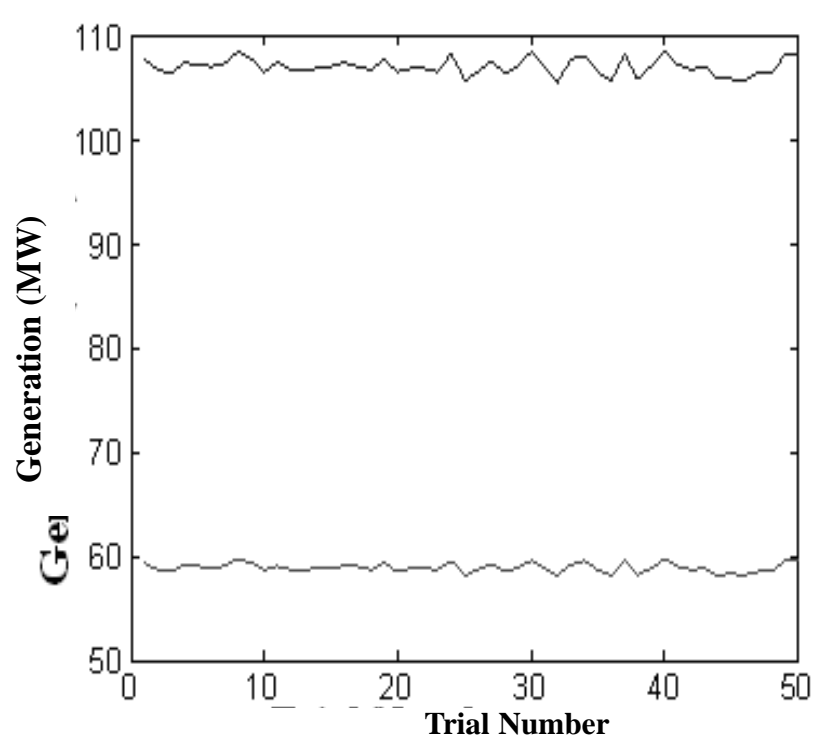

a) $G D$ group $\left(G_{1}, G_{2}\right)$

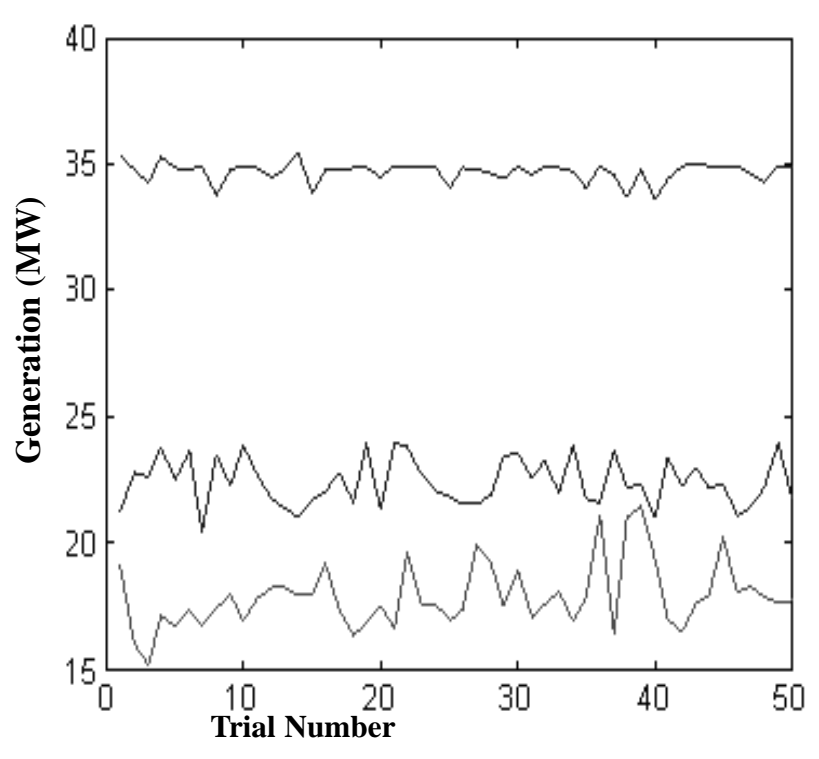

b) GI group $\left(G_{8}, G_{11}, G_{13}\right)$

Figure 7. Trial by trial statistical performance for outage of line 4-12

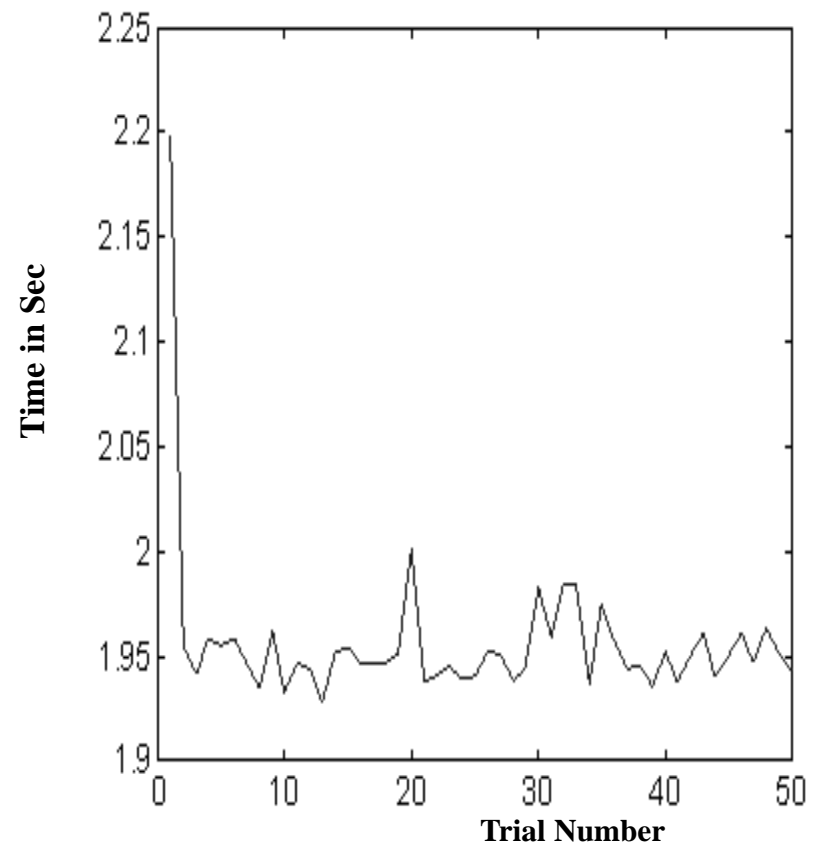

a) GD group

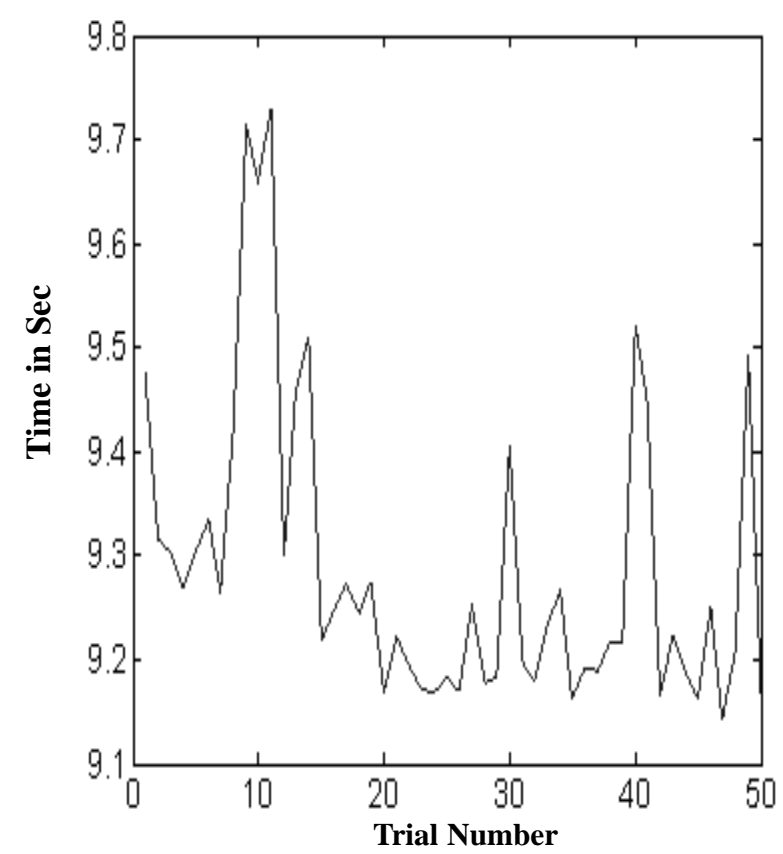

b) GI group

Figure 8. Statistical performance of convergence time for outage of line 4-12 
The Journal of Engineering Research Vol. 7, No. 1, (2010) 31-41

\subsection{Comparison study for overload of line 1-2 and 2-6}

In the IEEE 30 bus systems the lines 1-2 and 2-6 are overloaded. The generator area for the base case is given in Table-1, and the DAG is shown in Fig. 5a. The overload in the lines 2-6 is due to the load demand in the generator areas 5, 8 and 11. The lines 2-6 receive power from generator areas 1 and 2, so the $\mathrm{GA}_{1}$ and $\mathrm{GA}_{2}$ are declared as the GD group. The power flows through the lines 2-6 to supply the loads in the generator areas $5,8,11$ and $\mathrm{GA}_{\mathrm{Com}}$. Generator areas 5, 8, 11 and $\mathrm{GA}_{\mathrm{Com}}$ are declared as GI group. The lines 1-2 and 2-6 overloads are removed by rescheduling the generators $1,2,5,8$ and 11 . The comparison results for overload alleviation by generator rescheduling are given in Table 4.

Before rescheduling lines 1-2 and 2-6 are loaded to 170.04 MVA and 66.79 MVA with respect to their line limit 130 MVA and 65 MVA respectively. After rescheduling the power flows on the lines 1-2 and 2-6 are 129 MVA and 60 MVA reported in Dutta et al. (2008) whereas in the proposed approach they are found to be 129.35 MVA and 61.37 MVA respectively.

\subsection{Statistical Performance Analysis}

The GD and GI group optimizations are carried out for 50 independent trials for the IEEE 30 bus system. The maximum and minimum generation output, best output obtained, and the maximum number of times for the GD and GI groups, are given in Table 5.

The trail-by-trail statistical performance in respect of generation output and convergence times for the GD and GI groups for outage of lines 4-12 of the IEEE 30 bus system are shown graphically in Figs. 7 and 8 respectively.

From the above results, we observe that the proposed method can alleviate the line overloads due to contingency, in any system within minimal control actions, thereby preventing the cascading of outages, leading to blackout or system collapse.

\section{Conclusions}

A novel approach to corrective control strategy of generation-rescheduling and/or load shedding with subject to contingencies is presented. Identification of effective generator and/or load buses due to a contingency is achieved using a Direct Acyclic Graph (DAG). The concept of local optimization is utilized, wherein the implementation of control action becomes easy and effective. This enables the operator to quickly select the appropriate number of buses for a good sub-optimal solution. This task is achieved by means of a particle swarm optimization (PSO) method, which provides the best solution with less control decision and actions corresponding to generation and/or load increase/decrease respectively. The solution was sufficient for initiating control actions during an emergency as it protects the system from cascading outages.

\section{References}

Abou El-Ela, A.A., Bishr, M., Allam, S. And El-Sehiemy, R., 2005, "Optimal Preventive Control Actions using Multi-objective Fuzzy Linear Programming Technique," Electric Power Systems Research, Vol. 74, pp. 147-155.

Bhaskar, G. and Mohan, M.R., 2008, "Security Constrained Economic Load Dispatch using Improved Particle Swarm Optimization Suitable for Utility," Electric Power and Energy System, Vol. 30, pp. 609-613.

Bialek, J., 1996, "Tracing the Flow of Electricity," IEE Proceedings-Generation Transmission Distribution, Vol. 143(4), pp. 313 - 320.

Chan, S.M. and Schweppe, F.C., 1997, "Ageneration Reallocation and Load Shedding Algorithm," IEEE Transaction on Power Apparatus and Systems, Vol. PAS-98(1), pp. 26-34.

Christie,R.D., Wollenberg, B. and Wangensteen, I., 2000, "Transmission Management in the Deregulated Environment," Proceedings of the IEEE, Vol. 88(2), pp.170-195.

Dutta, S. and Singh, S.P., 2008 , "Optimal Rescheduling of Generators for Congestion Management Based on Particle Swarm Optimization", IEEE Transactions on Power Systems, Vol. 23(4), pp 1560-1569.

Hazra, J. and Sinha, A. K., 2007, "Congestion Management using Multi-objective Particle Swarm Optimization," IEEE Transaction on Power Systems, Vol. 22(4), pp. 1726 - 1734.

Kennedy, J. and Eberhart, R., 1995, "Particle Swarm Optimization," Proceeding of IEEE, International Conference in Neural Network, Perth, Austrlia, pp. 1942-1948.

Kirschen, D., Allan, R. and Strbac, G., 1997, "Contributions of Individual Generators to Loads and Flows," IEEE Transaction on Power Systems, Vol. 12(1), pp. 52-60.

Medicheral, T.K.P., Billinto, R. and Sachdev, M.S., 1981, "Generation Rescheduling and Load Shedding to Alleviate line Overloads - System Studies," IEEE Transaction on Power Apparatus and Systems, Vol. PAS-100(1), pp. 36-42.

PSO Tutorial. [Online]. Available: http://www.swarmintelligence.org/tutorials.php

Ram, D., Srivastava, L., Pandit, M. and Sharma, J., 2007, "Corrective Action Planning using RBF Neural Network," Applied Soft Computing, Vol. 7, pp. 10551063.

Shandilya, A., Gupta, H. and Sharma, J., 1993, "Method for Generation Rescheduling and Load Shedding to Alleviate Line Overloads using Local Optimization," IEE Proceedings Generation, Transmission and Distribution, Vol. 140(5), pp. 337-342.

Talukdar, B.K., Sinha, A.K., Mukhopadhyay, S. and Bose, A., 2005, "A Computationally Simple Method for 
The Journal of Engineering Research Vol. 7, No. 1, (2010) 31-41

Cost-efficient Generation Rescheduling and Load Shedding for Congestion Management," Electrical power and Energy Systems, Vol. 27, pp. 379-388.

Yesuratnam, G. and Thukaram, D., 2007, "Congestion Management in Open Access Based on Relative Electrical Distance using Voltage Stability Criteria," Electric Power System Research, Vol. 77, pp.1608 1618. 